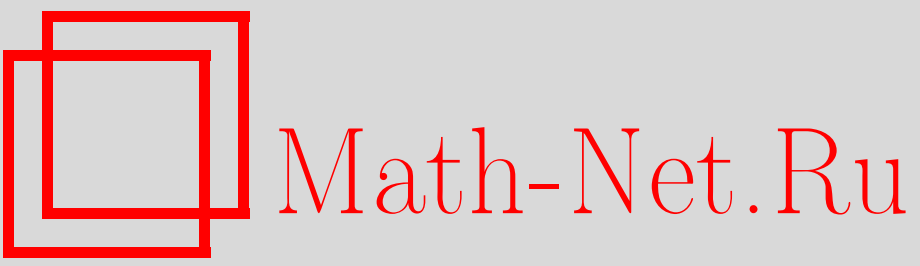

О. И. Богоявленский, Необходимые условия для разделимости систем уравнений в частных производных произвольного порядка, УМН, 2005, том 60, выпуск 1, 163-164

DOI: https://doi.org/10.4213/rm1394

Использование Общероссийского математического портала Math-Net.Ru подразумевает, что вы прочитали и согласны с пользовательским соглашением

http: //www . mathnet.ru/rus/agreement

Параметры загрузки :

IP: 35.174 .16 .151

26 апреля 2023 г., 14:37:57 


\section{НЕОБХОДИМЫЕ УСЛОВИЯ ДЛЯ РАЗДЕЛИМОСТИ СИСТЕМ УРАВНЕНИЙ В ЧАСТНЫХ ПРОИЗВОДНЫХ ПРОИЗВОЛЬНОГО ПОРЯДКА}

\section{О. И. БОГОявлЕнСКий}

В работе вьведены необходимые условия для разделимости общей системы уравнений в частных производных (УрЧП)

$$
\frac{\partial^{m} u^{i}}{\partial t^{m}}=\sum_{|\alpha|=K} \sum_{j=1}^{n} A_{\alpha j}^{i}(u) \frac{\partial^{K} u^{j}}{\partial x_{\alpha}}+F^{i}\left(u, \frac{\partial u}{\partial t}, \frac{\partial^{|\beta|} u}{\partial x_{\beta}}, \ldots, \frac{\partial^{m-1} u}{\partial t^{m-1}}, \frac{\partial^{K-1} u}{\partial x_{\gamma}}\right)
$$

на $q$ невзаимодействующих подсистем или на $q$ треугольно-взаимодействующих подсистем. Здесь $u^{i}=u^{i}\left(t, x_{1}, \ldots, x_{k}\right), i, j=1, \ldots, n$, и $\alpha, \beta$ являются мультииндексами, $\alpha=\left(i_{1}, \ldots, i_{k}\right),|\alpha|=$ $i_{1}+\cdots+i_{k}=K,|\beta|,|\gamma| \leqslant K-1$. Система (1) после преобразовани

$$
u^{i} \longrightarrow v^{j}=v^{j}\left(u^{1}, \ldots, u^{n}\right)
$$

принимает вид

$$
\frac{\partial^{m} v^{i}}{\partial t^{m}}=\sum_{|\alpha|=K} \sum_{j=1}^{n} \bar{A}_{\alpha j}^{i}(v) \frac{\partial^{K} v^{j}}{\partial x_{\alpha}}+\bar{F}^{i}\left(v, \frac{\partial v}{\partial t}, \frac{\partial^{|\beta|} v}{\partial x_{\beta}}, \ldots, \frac{\partial^{m-1} v}{\partial t^{m-1}}, \frac{\partial^{K-1} v}{\partial x_{\gamma}}\right),
$$

где

$$
\bar{A}_{\alpha j}^{i}(v)=\sum_{p, q=1}^{n}\left(D^{-1}\right)_{p}^{i} A_{\alpha q}^{p}(u) D_{j}^{q}, \quad D_{j}^{i}=\frac{\partial u^{i}}{\partial v^{j}} .
$$

ЗАмечАниЕ 1. Формула (3) означает, что для любых $m \geqslant 1$ и $K \geqslant 1$ и для любого мультииндекса $\alpha,|\alpha|=K$, коэффициенты $A_{\alpha j}^{i}(u)$ образуют $(1,1)$-тензоры по отношению к преобразованиям (2). Это свойство систем (1) УрЧП произвольного порядка обобшает хорошо известное свойство [1] систем первого порядка, для которых $m=K=1$.

Для каждого $(1,1)$-тензора $A_{\alpha j}^{i}(u)$ мы используем соответствующий $(1,2)$-тензор Нийенхейca [2]:

$$
N_{\alpha j k}^{i}=\sum_{p=1}^{n}\left(\frac{\partial A_{\alpha k}^{i}}{\partial u^{p}} A_{\alpha j}^{p}-\frac{\partial A_{\alpha j}^{i}}{\partial u^{p}} A_{\alpha k}^{p}+\frac{\partial A_{\alpha j}^{p}}{\partial u^{k}} A_{\alpha p}^{i}-\frac{\partial A_{\alpha k}^{p}}{\partial u^{j}} A_{\alpha p}^{i}\right),
$$

который определяет кососимметрическое векторное произведение любых двух касательных векторов $V, U \in T_{u}\left(\mathbb{R}^{n}\right): N_{\alpha}(V, U)=-N_{\alpha}(U, V) \in T_{u}\left(\mathbb{R}^{n}\right)$.

ОПредЕлЕниЕ. Для любых касательных векторов $V \in T_{u}\left(\mathbb{R}^{n}\right)$, рассмотрим линейные преобразования $N_{\alpha V}$ в касательных пространствах: $U \longrightarrow N_{\alpha V}(U)=N_{\alpha}(V, U)$, где $U \in T_{u}\left(\mathbb{R}^{n}\right)$. Определим инвариантные многочлены от компонент вектора $V$ :

$$
P_{K}(V)=\operatorname{det}\left\|B_{K V}-Q\right\|, \quad B_{K V}=\sum_{|\alpha|=K} Q_{\alpha} N_{\alpha V},
$$

где операторы $Q=Q\left(A_{\delta}, \ldots, A_{\tau}\right)$ и $Q_{\alpha}=Q_{\alpha}\left(A_{\delta}, \ldots, A_{\tau}\right)$ являются произвольньми многочленами от операторов $A_{\delta}, \ldots, A_{\tau},|\delta|=\cdots=|\tau|=K$.

Покажем, что многочлен $P_{K}(V)$ имеет степень $\operatorname{deg} P_{K}(V) \leqslant n-1$. Действительно, оператор $B_{K V}(5)$ линейно зависит от компонент вектора $V$. Сумма всех слагаемых наивысшей степени $n$ в многочлене $P_{K}(V)$ равна $\operatorname{det}\left\|B_{K V}\right\|$. Однако $\operatorname{det}\left\|B_{K V}\right\| \equiv 0$, потому что $B_{K V}(V)=0$, поскольку $N_{\alpha V}(V)=N_{\alpha}(V, V)=0$. Поэтому $\operatorname{deg} P_{K}(V) \leqslant n-1$. 
Теорема 1 (необходимое условие для разделимости). Если система (1) может быть разделена на $q \geqslant 2$ невзаимодействующих подсистем, то многочлен $P_{K}(V)$ (5) имеет степень $\operatorname{deg} P_{K}(V) \leqslant n-q$.

ДокАЗАТЕльСтво. Предположим, что в некоторых координатах $v^{1}, \ldots, v^{n}$ система (1) разделяется на $q \geqslant 2$ невзаимодействующих подсистем размерностей $m_{1}, \ldots, m_{q}$, где $m_{1}+\cdots+$ $m_{q}=n$. Тогда соответствующие $(1,1)$-тензоры $A_{\alpha j}^{i}(v)$ (и операторы $Q, Q_{\alpha}$ ) в координатах $v^{1}, \ldots, v^{n}$ имеют вид $q$ диагональных блоков $A_{\alpha 1}(v), \ldots, A_{\alpha q}(v)$ размеров $m_{1} \times m_{1}, \ldots, m_{q} \times$ $m_{q}$, которые зависят от различных групп переменных $v^{i}$. Соответствующие тензоры Нийенхейса $N_{\alpha j k}^{i}(4)$ являются прямшти суммами тензоров Нийенхейса $N_{\alpha \ell}$ в подпространствах $\mathbb{R}^{m_{\ell}}$, где $\ell=1, \ldots, q$. Поэтому многочлен $P_{K}(V)$ имеет вид

$$
P_{K}\left(V_{1}+\cdots+V_{q}\right)=\operatorname{det}\left\|\sum_{|\alpha|=K} Q_{\alpha} N_{\alpha V}-Q\right\|=P_{K 1}\left(V_{1}\right) \cdots P_{K q}\left(V_{q}\right) .
$$

Каждый многочлен $P_{K}\left(V_{\ell}\right)$ имеет степень $\leqslant m_{\ell}-1$. Поэтому степень многочлена $P_{K}(V)$ удовлетворяет соотношению $\operatorname{deg} P_{K}(V) \leqslant n-q$.

ЗАмечаниЕ 2. Другое необходимое условие получается путем применения в формуле (5) $(1,2)$-тензора Хаантжеса $H_{\alpha j k}^{i}$ [3] вместо тензора Нийенхейса $N_{\alpha j k}^{i}(4)$. Необходимое условие для полного разделения системы (1) на $n$ невзаимодействующих уравнений имеет вид: для любого многочлена $Q\left(A_{\delta}, \ldots, A_{\gamma}\right)$ с постоянньми коэффициентами соответствующий тензор Нийенхейса $N_{Q j k}^{i} \equiv 0$.

Система УрЧП (1) имеет $q$ треугольно-взаимодействующих подсистем, если в некоторых координатах $v^{1}, \ldots, v^{n}$ система (1) имеет замкнутую подсистему в подгруппе переменных $v^{1}, \ldots, v^{m_{1}}$, которая содержится в замкнутой подсистеме в переменных $v^{1}, \ldots, v^{m_{1}}$, $v^{m_{1}+1}, \ldots, v^{m_{1}+m_{2}}$ и т. д., где $m_{1}+\cdots+m_{q}=n$.

ТеОРема 2. Если система (1) имеет $q \geqslant 2$ треугольно-взаимодействующих подсистем, то выполняется необходимое условие для многочлена (5): $\operatorname{deg} P_{K}(V) \leqslant n-q$. Если система (1) имеет замкнутую собственную подсистему, то $\operatorname{deg} P_{K}(V) \leqslant n-2$.

Доказательство аналогично доказательству теоремы 1.

ПримеР. Система Бенни для $2 \ell$ функций $u^{i}(t, x), \eta^{i}(t, x), i=1, \ldots, \ell$, имеет вид

$$
u_{t}^{i}=-u^{i} u_{x}^{i}-\left(\eta_{1}+\cdots+\eta_{\ell}\right)_{x}, \quad \eta_{t}^{i}=-\left(\eta^{i} u^{i}\right)_{x} .
$$

Соответствующий многочлен $P_{K}(V)(5)$, где $K=1, k=1$ и $Q=Q_{\alpha}=1$, определяется формулой

$$
P_{1}(V)=\left(V^{\ell+1}+\cdots+V^{2 \ell}-1\right)^{2 \ell-1},
$$

где $V=V^{1} \partial_{u_{1}}+\cdots+V^{\ell} \partial_{u_{\ell}}+V^{\ell+1} \partial_{\eta_{1}}+\cdots+V^{2 \ell} \partial_{\eta_{\ell}}$. Многочлен $P_{1}(V)$ (7) имеет максимально возможную степень $n-1, n=2 \ell$. Поэтому, применяя теорему 2 , мы получаем, что система Бенни (6) не имеет замкнутых собственных подсистем и, следовательно, неразложима.

\section{СПИСОК ЛИТЕРАТУРЫ}

[1] A. P. Stone // Proc. Amer. Math. Soc. 1967. V. 18. P. 868-873. [2] A. Nijenhuis // Nederl. Akad. Wetensch. Proc. Ser. A. 1951. V. 13. P. 200-212. [3] J. Haantjes // Nederl. Akad. Wetensch. Proc. Ser. A. 1955. V. 17. P. 158-162.

Математический институт им. В. А. Стеклова РАН, Queen's University, Kingston, Canada
Представлено В.М. Бухштабером Принято редколлегией 23.11.2004 